\title{
Simple Approximation of the Magnetic Induction Inside High Voltage Overhead Transmission Lines
}

\author{
Salah-Eddine Houicher ${ }^{1, *}$, Rabah Djekidel ${ }^{1}$, and Sid-Ahmed Bessidik ${ }^{1}$ \\ ${ }^{1}$ Ammar Telidji University, Electrical Engineering Department, LACoSERE Laboratory, 03000 Laghouat, Algeria
}

\begin{abstract}
With the development of electrical power transmission networks, are resulted the increase and continuous consumption of various power production sources, this is what requires the large quantities of energy construction. The increased demand in electric power employment has generated an raised of operating voltage for transmission lines (HV and VHV). The high voltage overhead transmission lines create electric and magnetic fields, and therefore have elevated serious concern questions about their possible potential effects in human body and environmental great associated with the resulting higher levels of electromagnetic fields strength around these power lines. The accurate evaluation of extremely low frequency electromagnetic fields influences produced by the high voltage overhead power lines on human health and the environment are well very known and analysed in several research works and simulation study projects. In this paper we precise examine and present a methodology for plot the lateral profile of magnetic field distribution in close a three-phase overhead transmission line in single circuit flat horizontal configuration at height $1 \mathrm{~m}$ above the ground level, In order show the essentials factors affecting in the magnetic fields intensity under and in the vicinity of high voltage overhead transmission line in any point of space surface and ground plane, the analytique calculation results of the lateral profile of magnetic flux density behaviour are obtained by program MATLAB software, which makes it possible better analyser and easy represent the transverse profile of the magnetic field around electric power transmission lines, the numerique simulation results are based in image method and the superposition theorem.
\end{abstract}

keywords: overhead power line; magnetic field; lateral profile; exposure limit; power frequency; simulation; sag conductor.

\section{1 introduction}

The improvement of high voltage electrical power plants causes the problems related to electromagnetic fields produces the interactions with near objects and diverse problems [1]. High fields levels that can cause damage to the environment including substances located nearby these networks [2], moreover they could expose the populates working around them to serious diseases. Over the years, electric power has better the conditions of human living, it plays a key role in responding to basic human being needs. However, despite all its advantages, electricity has many negative effects on human health and the environment. As energy needs increase with the rapid advance of the human population, principal to adoption of electric power transmission systems with very high voltage levels and augmented the creation of new circuit power lines using single or double circuit lines near residential areas [3]. The electromagnetic fields of extremely low frequency(ELF) generated by high voltage transmission lines have assumed great importance in recent years, widely study by the scientific community because of concerns existed by government agencies, non-governmental and energy utilities about their possible biological effects on health and the environment, exposure to these electromagnetic fields induces a current inside human body that interferes with those, and can if necessarily intense, causes harmful biological effects with important implications for human health [4]. The increase in the population over the world drive promote the energy needed which reasons the evolution of the electric power demand and speed up the concentration of transmission lines with a high operating voltage levels. These transmission lines engender electric and magnetic fields, therefore advanced harmful questions about the potential health and environmental effects related with high levels of electromagnetic fields intensity around these circuit lines. in the last years, several research publications have been made for the simulation and measurement of very low frequency electric and magnetic fields (ELF) created by electric power transmission lines [5], Most adopt that the power lines are horizontal straight parallel to the ground, and the sag due to the power lines weight is

\footnotetext{
* Corresponding author: houichersalah@gmail.com
} 
neglected or introduced by taking an average height between the maximum and minimum height of the power lines. Based on the results and recommendations reported by these research studies. A number of national and international standards have been established, to define the exposure limits values for occupational and general public of electric and magnetic fields at very low frequency [6]. It is well decided by the World Health Organization (WHO) that these currents excite less effects reversible that are more visible from the current density of $10 \mathrm{~mA} / \mathrm{m}$. The limits of exposure to electromagnetic fields (EMF) generated by overhead power lines are resulting from the International Commission on Non -Ionizing Radiation Protection (ICNIRP). At low frequency $50 \mathrm{~Hz}$, the reference levels for general public exposure are $100 \mu \mathrm{T}$, and $500 \mu \mathrm{T}$ for occupational exposure (magnetic induction), in the other hand, $5 \mathrm{kV} / \mathrm{m}$ applied to the general public, and $10 \mathrm{kV} / \mathrm{m}$ for professional exposure (electric field) respectively. The reference levels for occupational exposure are $1 \mathrm{mT}$ and $10 \mathrm{kV} / \mathrm{m}$ [7]. These limit values are sometimes approached in areas close to huge transmission lines. With the increase power demand, the electric transmission utilities are increased the function voltage levels for effective magnitude power transmission. With the increase in operating voltage levels, high voltage overhead transmission lines carry bulk amounts of power and in turn large currents, therefore are expected to produce high values of magnetic fields. Consequently, in the design of extra high voltage overhead electric power lines, one of the important factors to be considered is the magnetic field distribution at the ground level or at height $1 \mathrm{~m}$ above ground surface. Precise calculation of the magnetic flux density values under overhead power lines is important from the view point of environmental impact. It is well known that the geometrical parameters of overhead power transmission lines (OPLs) affect the magnetic field values and profiles under and around them. Previous parameters effect on the magnetic field intensity at any point on the ground surface or in the space under and in the vicinity of overhead power circuit lines, such as the height of conductors, their size and phase conductors spacing together with the location of observed point where the field is to be calculated [8]. The loading currents also affect the magnitude of components. Theorical formulas were given for predicting the magnetic fields of power lines given knowledge of the currents in power line conductors beside the circuit lines configuration [9]. The analytical results of the magnetic field calculation of a very high voltage single circuit line based on image method using MATLAB numerical software which makes it possible to better analyse and to represent two dimension plot 2D transverse profile of the magnetic field distribution under the high voltage overhead power line. Nowadays, many laboratory experiments and literature lectures in the electrical engineering around the world are being assisted by computer simulations [10-11]. The modulation of electrical power systems has acquired great importance in engineering education because of the huge increase of the computer importance based systems in learning methodologies. The present article contracts with the Matlab package because it is widely used in electrical engineering courses [12-13]. It is well known that processes related to generation, transmission, distribution, and consumption of electric power plants are associated with the generation of low-frequency electromagnetic fields $(50-60 \mathrm{~Hz})$ and harmonic frequencies). The constant increase in the world's demand for electricity requires the permanent construction of new circuit lines and the extension of the existing transmission and distribution power lines. Thus, it is very important that electrical engineers obtain knowledge about the possible effects of electric and magnetic fields as well as providing them tools for computing and predicting the electric field intensity and magnetic flux density in the vicinity of high-voltage overhead power lines. In this work a fast method to simulate the magnetic fields created by single circuit three-phase power lines is presented, including the effects of a conducting ground and the effects of sagging conductors. The objective of the proposed system is the interpretation of a standard executable program's output results, also understand the physical and electrical laws involved in the computation of magnetic field at $1 \mathrm{~m}$ above the ground under overhead power line, in order show the numerous factors affecting in the magnetic induction values. understanding the steps involved in the computation of the magnetic field generated by overhead power lines. The methodology proposed for computing the magnetic field has been used by the authors of this work, in the other hand the simulation results with experimental data and alternative computing methods reported by Garrido and al [14], resulting a close similitude between them. Note that most references that deals with the magnetic field computation in the vicinity of overhead power lines [14-16] do not take into account the catenary shape of sagging conductors. These studies suppose that the overhead conductors are perfectly flat, thereby the geometry problem is greatly simplified. This simplification leads to a problem with a high degree of symmetry. Thus, under these conditions the Ampere's law can be applied, simplifying significantly the problem which is the object of this study. Moreover, so far a detailed study of the magnetic field generated by sagging conductors with catenary shape has not been reported for educational purposes. This paper is organized into three sections, Section II describes the theorical formulas used for calculation of power line magnetic fields based in Garson method and the superposition principle. Computation results of numerique simulation are offered and discussed in Section III. Section IV is conclusions.

\section{Computation of the magnetic field under high voltage overhead lines}

Although the electricity supply generally has electric and magnetic fields associated with it, high-voltage power lines are a particular source of exposure to elevated levels of these fields. The electrical and magnetic fields will be decoupled, that is to say, they will be independent of each other. This indicates that one cannot be deduced from the other but rather that they have to be 
calculated separately. It is also necessary to take into account that the electrical field is due to the difference in voltage between the power line and the ground and that the magnetic field is generated by the current transported by the line. In this work study depicts a cross-sectional view of a single-circuit three phase overhead power line $(400 \mathrm{kV}, 1.4 \mathrm{GVA})$ that will be used here for exemplification purposes. The phase conductors are arranged in a flat configuration, each phase comprising a bundle of two sub conductors (L1, L2, L3). A pair of two ground wires (G1-G2) is also represented in detail. Assuming that the overhead phase bundles are replaced with equivalent single conductors; assuming these conductors carry sinusoidal currents directed along the axis, with complex amplitudes (phasors) denoted by $\mathrm{I}_{1}, \mathrm{I}_{2}$ and $\mathrm{I}_{3}$, and assuming no other currents are present, the complex amplitude of the magnetic induction field vector in a point $1 \mathrm{~m}$ above ground is obtained, from Ampère's law, as a simple summation of three terms of the magnetic field components in overhead power line.

\subsection{Original field of the line}

The process starts with the given line geometry and with its specified loading conditions. In our case, phase bundles are located at:

$\mathrm{x} 1=-15.0[\mathrm{~m}], \mathrm{y} 1=20.0[\mathrm{~m}]$ for phase 1

$\mathrm{x} 2=0.0[\mathrm{~m}], \mathrm{y} 2=20.0[\mathrm{~m}]$ for phase 2 , and

$\mathrm{x} 3=15.0[\mathrm{~m}], \mathrm{y} 3=20.0[\mathrm{~m}]$ for phase 3

The Corresponding phase currents are:

$$
\begin{aligned}
& I_{1}=1500 \angle 0^{\circ}[\mathrm{A}] \\
& I_{2}=1500 \angle-120^{\circ}[\mathrm{A}] \\
& I_{3}=1500 \angle 120^{\circ}[\mathrm{A}]
\end{aligned}
$$

In the first step, the ground-level magnetic field caused by phase currents is computed. The general approach follows Ampere's Law as illustrated by Fig. 1. A current $I_{\mathrm{i}}$ at the point $\left(\mathrm{x}_{\mathrm{i}}, \mathrm{y}_{\mathrm{i}}\right)$ causes magnetic field intensity at location $\left(\mathrm{x}_{\mathrm{j}}, \mathrm{y}_{\mathrm{j}}\right)$ of the magnitude definite by this formula:

$$
\left|H_{i j}\right|=\frac{I_{i}}{2 . \pi \cdot r_{i j}}\left[\frac{A}{m}\right]
$$

\subsubsection{Calculation of the magnetic field generated by an Infinite and Isolated straight conductor}

Mostly high-voltage power lines use a three-phase system in order to transport electric energy. This means that they consist of three conductors with alternating sinusoidal voltages of $50 \mathrm{~Hz}$ with equal amplitude but out of phase with each other at an electric angle of $120^{\circ}$. Therefore, this angle must be taken into account in the calculation of the magnetic field. In this section a formula to determine the magnetic field generated by an infinite and isolated straight conductor is deduced. Fig. 1 shows a straight conductor along which a current $I_{i}$ circulates, which generates a magnetic field $B_{i}$ in the space that surrounds it. The magnetic field generated by an infinite straight conductor placed in position $\left(\mathrm{x}_{\mathrm{i}}, \mathrm{y}_{\mathrm{i}}, \mathrm{Z}_{\mathrm{i}}\right)$ can be determined by applying Biot Savart law [17],

$$
d \vec{B}_{i}=\frac{\mu_{0} I_{i}}{4 \pi} \frac{d \vec{l} \wedge \vec{r}}{r^{3}}
$$

being $\vec{r}=\left(x-x_{i}\right) \hat{i}+\left(y-y_{i}\right) \hat{j}+0 \hat{k}, \quad \mu 0=4 \pi \times 10^{-7}$ $\mathrm{N} / \mathrm{A}^{2}$, and $d \vec{l}=d z \hat{k}$ has the same direction as $I_{i}$. Therefore, as deduced in Ref. [17] the result is

$$
B_{i}=\frac{\mu_{0}}{2 \pi_{i}} \frac{I_{i}}{r_{i}}
$$

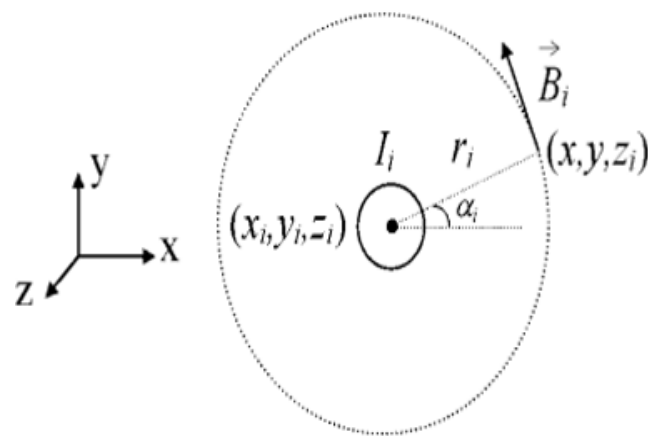

Fig. 1. Vectorial magnetic field created by a single conductor.

From both, Fig. 1 and Equation (4), it can also be deduced that the vectorial magnetic field expression is given by the following formula:

$\vec{B}_{i}=\frac{\mu_{0} I_{i}^{\varphi}}{2 \pi}\left(\frac{\left(y-y_{i}\right)}{\left(x-x_{i}\right)^{2}+(y-y i)^{2}}, \frac{\left(x-x_{i}\right)}{\left(x-x_{i}\right)^{2}+\left(y-y_{i}\right)^{2}}, 0\right)$

Where $\varphi$ is the phase angle of the current flowing in the phase conductors of electric power line. It is necessary to take into account that $I_{i}$ and $\mathrm{B}_{\mathrm{i}}$ are sinusoidal alternating physical variables, and it is convenient to express their magnitudes in the root mean square (RMS) value.

\subsubsection{Image methodology principle}

The image method is helpful to take into account the impacts of the presence of a conducting ground [18]. The alternating magnetic field that the line generates induces return currents in thd ground and these, at the same time generate a magnetic field that is composed to that produced by the line. The image theory states that the field created by a current carrying conductor, when placed at height $D_{\text {erc }}$ above a perfectly conducting ground, can be represented by the common fields of conductors and its image [19]. When the finitely conducting ground is replaced with a perfectly conducting ground, standard image theory can be used to locate the conductor image depth. As depicted in Fig. 2, the image conductor is placed at a depth given by $\mathrm{D}_{\text {erc }}+\mathrm{y}_{\mathrm{i}}$, being $\mathrm{D}_{\text {erc }}$ a complex distance [20] which value depends on the ground electrical conductivity as expressed in Equation (6). 


$$
D_{e r c}=658.87 \cdot \sqrt{\frac{\rho_{s}}{f}}
$$

Where, $\rho_{\mathrm{s}}$ is the electrical resistivity of the earth expressed as $\Omega . \mathrm{m}, f$ is the frequency of the source current in $\mathrm{Hz}$. In the case of a perfectly conducting ground it results $\delta=0$ and, therefore $\alpha=0$. However, terrains of low conductivity give a very large penetration depth $\delta$ and, therefore, $\alpha$ is also very high. In the case of a perfect insulator the result is $\alpha=\infty$.

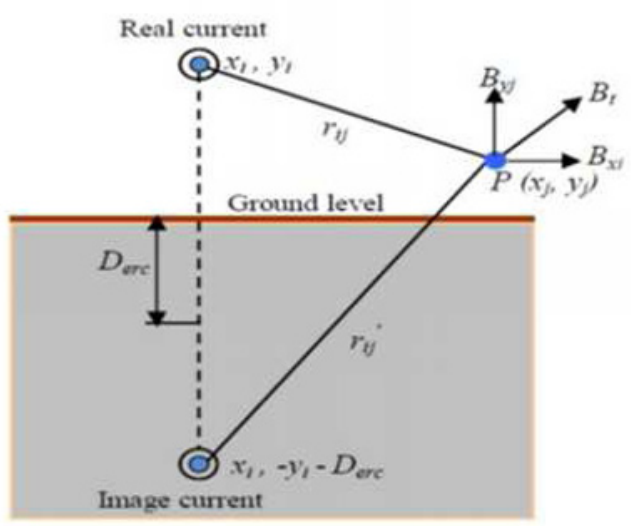

Fig. 2. Magnetic field engender by reel current and its image.

Practical ground resistivities fluctuate from vary from $\rho<50 \Omega$.m for especially conducting ground to $\rho>3,000 \Omega . m$ for very poor conducting ground [21]. In order to calculate the resistance of ground, $\rho=100 \Omega . \mathrm{m}$ has been used. By substituting in Equation (6) $\mathrm{f}=50 \mathrm{~Hz}$, $\rho=100 \Omega$.m, and $\mu_{0}=4 \pi \times 10^{-7}$ gives a calculation result of $\delta=711.76 \mathrm{~m}$. This value is approach superior to the distance between overhead current carrying conductors and the ground. The components of magnetic flux density due to a conductor are given by the two components vertical and horizontal in the equations above. The deviation of the normal component of the magnetic field strength from the zero value is calculated at a set of check points (match points) chosen on the sub-conductor's surfaces, the values and positions of simulation currents are known, the distribution of the magnetic field in any region can be calculated easily. Since the typical value for the conductivity of the earth is in range from 0.001 to $0.1 \mathrm{~S} / \mathrm{m}$, the image currents are located at hundreds of meters below the ground. This image expression is valid under two conditions: if the frequency is low and if the ground is nonmagnetic. The horizontal and vertical components of the magnetic flux density around high voltage overhead power line can be calculated by following [22-23]:

$$
\left.\begin{array}{l}
B_{x j}=-\frac{\mu_{0}}{2 . \pi} \cdot I_{n}\left[\frac{y_{i}-y_{j}}{r_{i j}{ }^{2}}-\frac{y_{i}+y_{j}+D_{e r c}}{r_{i j}{ }^{\prime 2}}\right] \\
B_{y j}=\frac{\mu_{0}}{2 . \pi} \cdot I_{n}\left[\frac{x_{i}-x_{j}}{r_{i j}{ }^{2}}-\frac{x_{i}-x_{j}}{r_{i j}{ }^{\prime 2}}\right]
\end{array}\right\}
$$

Where, $\left(\mathrm{x}_{\mathrm{i}}, \mathrm{y}_{\mathrm{i}}\right)$ and $\left(\mathrm{x}_{\mathrm{j}}, \mathrm{y}_{\mathrm{j}}\right)$ are the coordinates of the observation point and location of simulation line current, respectively; $r_{i j}$ is the distance between each conductor and observation point above ground, $\mathrm{r}_{\mathrm{ij}}{ }^{\prime}$ is the distance between each image conductor and observation point.

The magnetic field of transmission line can be written in adding the components of the given field for each conductor:

$$
\left.\begin{array}{c}
B_{x j}=\sum_{n=1}^{N} B_{x j} \\
B_{y j}=\sum_{n=1}^{N} B_{y j}
\end{array}\right\}
$$

The magnitude of the total magnetic induction at any desired point $\mathrm{P}$ is calculated by the summation of the horizontal and vertical components as a following:

$$
B_{t o t}=\sqrt{B_{x j}{ }^{2}+B_{y j}{ }^{2}}
$$

\subsection{Geometric and characteristic of line study}

We consider a high voltage three-phase overhead power line, Each phase of the line (L1, L2, L3) contains a bundle of two conductors in single circuit horizontal configuration parallel to the flat ground, are separated by $0.5 \mathrm{~m}$ between them, the radius of conductor is $1.16 \mathrm{~cm}$. The total section of a sub-conductor equals $590 \mathrm{~mm}^{2}$. The diameter of a sub-ray of sub-conductor is $23.2 \mathrm{~mm}$,

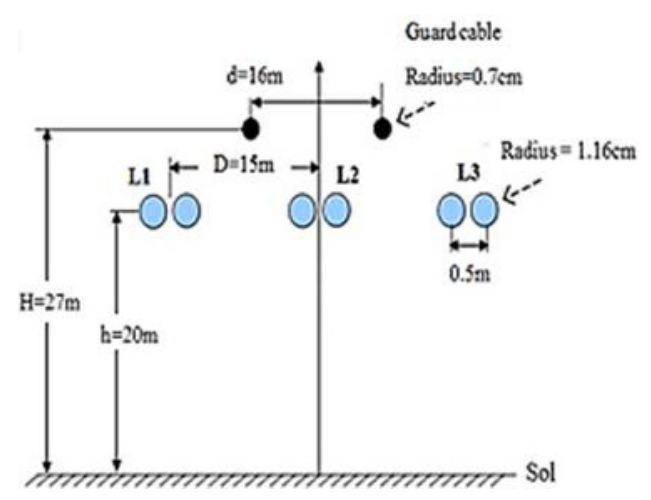

Fig. 3. Single circuit high voltage overhead transmission line.

While the guard cable is of wire having a single bundle of linear loads. the phase conductors are arranged on the same horizontal plane, that is to say the same height of conductors above the ground. This plant type it is usually used for electric power high voltage transmission lines. The conductors are at a height of $20 \mathrm{~m}$, the separation distance between phase conductors is $15 \mathrm{~m}$, the radius of guard cable $0.7 \mathrm{~cm}$ positioned horizontally at $-8 \mathrm{~m}$ and $+8 \mathrm{~m}$, at height $27 \mathrm{~m}$ above the ground, the span length of the power line is of $300 \mathrm{~m}$. The geometry of three-phase high voltage overhead transmission line of its characteristics and geometric coordinates presented in Fig.3. shows above, its parameters are listed in Table 1.

Table 1. Parameters used for overhead power circuit line. 


\begin{tabular}{|c|c|}
\hline Parameter & Value(m) \\
\hline $\begin{array}{c}\text { Height of } \\
\text { conductors }\end{array}$ & 20 \\
\hline $\begin{array}{c}\text { Spacing between } \\
\text { phase conductors }\end{array}$ & 15 \\
\hline $\begin{array}{c}\text { Height of the guard } \\
\text { cable above ground }\end{array}$ & 27 \\
\hline $\begin{array}{c}\text { Spacing between } \\
\text { guard cable }\end{array}$ & 8 \\
\hline $\begin{array}{c}\text { Spacing between } \\
\text { subconductors }\end{array}$ & 0.5 \\
\hline Span lenght & 300 \\
\hline
\end{tabular}

\section{Results and discussions}

For the simulation computation, are obtained for a balanced system in which the currents in phase conductors are symmetric and in direct succession at a phase angle $\varphi$ of $120^{\circ}$ to one another. For the power line situation, the magnitude of electric current circulates in the power line is $\mathrm{I}=1500 \mathrm{~A}$, the current through the guard cable is nul, $\mathrm{Ig}=0$. The resistivity of soil is considered as $\rho=100 \Omega$.m, and the source frequency $50 \mathrm{~Hz}$ are used for the numerical calculation of the magnetic flux density. The circuit line is assumed to carry the same amount of current magnitude, and earth wires effects on the circuit line are neglected. The effect of transmission line current unbalance is not considered, because it is usually low. The lateral profile distribution of magnetic field intensity depends on the phases arrangement. Underneath power lines the same phases arrangement produces the highest magnetic field; the magnetic flux density can be reduced either underneath or far from the lines by changing the phases arrangement. The currents system in the phase conductors is considered symmetrical and in direct positive succession as a following:

$$
\underline{I}_{L 1}=I, \underline{I}_{L 2}=I . e^{-j \frac{2 . \pi}{3}}, \underline{I}_{L 3}=I . e^{+j \frac{2 . \pi}{3}}
$$

In this case, from the simulation of magnetic field intensity, we found the following results present below:

Fig.4 illustrates the curve of the lateral profile distribution of horizontal, vertical and total components $(\mathrm{Bx}, \mathrm{By}, \mathrm{Bt}$ ) of the magnetic field at height $1 \mathrm{~m}$ above the ground level in the vicinity of three-phase high voltage overhead power line, as a function of the lateral distance of circuit line, according on the numerique simulation, it is very clear the magnetic field strength increases in symmetrically and continuous manner in the two sides positive and negative of the high voltage overhead transmission line in single circuit flat horizontal configuration, then decreases rapidly for significative increases of the lateral distance of overhead power line.

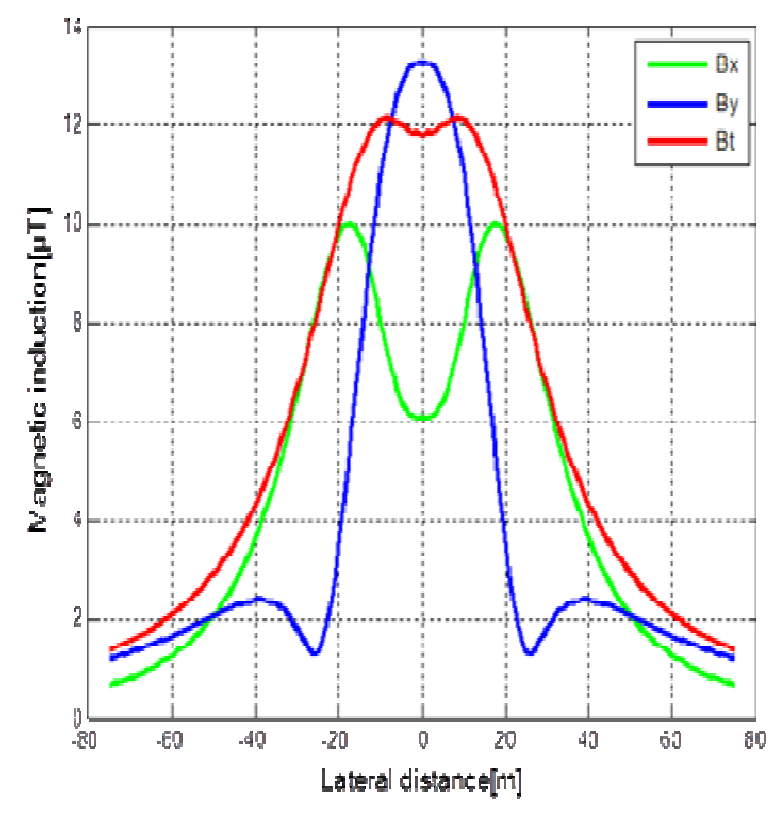

Fig. 4. Lateral profile of the magnetic induction distribution in $1 \mathrm{~m}$ above the ground level under overhead transmission line.

On observe in this curve graph, For the horizontal magnetic component $\mathrm{Bx}$, in the $\mathrm{x}$-axis of single circuit overhead power transmission line, the maximum value of lateral profile of the magnetic induction distribution is $\mathrm{Bmax}=10.03 \mu \mathrm{T}$ is registered in the distance located between the lateral and central conductor $\mathrm{x}=18$ meters near under the side phase conductor accounting for only $10.03 \%$ of the exposure limit established by ICNIRP for the general public $100 \mu \mathrm{T}$, then it slowly reduces until to register the minimum value $\mathrm{Bmin}=6.06 \mu \mathrm{T}$ in the symmetry point (center distance $\mathrm{x}=0$ meter) accounting for only $6.06 \%$ of the exposure limit established by ICNIRP for the general public $100 \mu \mathrm{T}$, is less intense under the middle phase conductor from where in this point it increases slowly again in symmetric manner in the two sides positive and negative of the high voltage overhead transmission line, until to reach the peak maximum intensity value of the magnetic induction $B \max =10.03 \mu \mathrm{T}$ near under the side phase conductor at distance located between the lateral and central conductor $x=18$ meters, near under the side phase conductor accounting for only $10.03 \%$ of the exposure limit established by ICNIRP for the general public $100 \mu \mathrm{T}$, then it slowly reduces until to register the minimum value $\mathrm{Bmin}=6.06 \mu \mathrm{T}$ in the symmetry point (center distance $\mathrm{x}=0$ meter) accounting for only $6.06 \%$ of the exposure limit established by ICNIRP for the general public $100 \mu \mathrm{T}$, is less intense under the middle phase conductor from where in this point it increases slowly again in symmetric manner in the two sides positive and negative of the high voltage overhead transmission line, until to reach the peak intensity value of the magnetic induction $\mathrm{Bmax}=10.03 \mu \mathrm{T}$ near under the side phase conductor at distance located between the lateral and central conductor $x=18$ meters, then decreases rapidly with significative increase of the lateral distance 
of single circuit line, to recorder the lower values when one moves away from the conductors, to reach at a lateral distance of 75 meters far from the center line a very low value is about $0.67 \mu \mathrm{T}$ almost negligible accounting for only $0.67 \%$ of the exposure limit established by ICNIRP for the general public $100 \mu \mathrm{T}$ approximately accounting ten times less than the maximum value that found under the line. For the vertical magnetic component By, in the $\mathrm{y}$-axis of single circuit transmission line, we find that unlike the electric field, the maximum value of lateral profile of the magnetic induction distribution $\mathrm{Bmax}=13.29 \mu \mathrm{T}$ which occurs at the center distance of power line (the symmetry point $\mathrm{x}=0$ meter), in the middle of the right-of-way transmission line(the center of phases), accounting for only $13.29 \%$ of the exposure limit established by ICNIRP for the general public $100 \mu \mathrm{T}$, which is takes the values max than that register at the same level as a comparing in the horizontal magnetic component ( $\mathrm{x}$ axis), then from this point the field it is strongly attenuated laterally rapidly in a continuous and symmetrically manner in two sides positive and negative of the single circuit line to reach a low value about $1.30 \mu \mathrm{T}$ accounting for only $1.30 \%$ of the exposure limit established by ICNIRP for the general public $100 \mu \mathrm{T}$ at a lateral distance of 26meters, elsewhere which the magnetic induction increases slightly again to register the minimum value about $2.39 \mu \mathrm{T}$ at a lateral distance about 39meters, after from this point the magnetic field begins also decreases rapidly in symmetric manner as a the lateral distance increases, to recorder the lowest values if when one moves away from the conductors, to reach at a lateral distance of 75 meters far from the center power line a minimum value approximately about $1.19 \mu \mathrm{T}$ almost negligible accounting for only $1.19 \%$ of the exposure limit established by ICNIRP. for the general public $100 \mu \mathrm{T}$, about ten times lower than the maximum value found under the line. On the other hand, On observe in this curve for the total magnetic component $\mathrm{Bt}$, the maximum value of lateral profile of the magnetic induction distribution $\mathrm{Bmax}=12.12 \mu \mathrm{T}$ is registered in the side phases conductor at a lateral distance located between the lateral and central conductor about $\mathrm{x}=9$ meters, accounting for only $12.12 \%$ of the exposure limit established by ICNIRP for the general public $100 \mu \mathrm{T}$, then reduces little in the center point of power line $(x=0$ meter) under the middle phase conductor, which is register a minimum value $\mathrm{Bmin}=$ $11.82 \mu \mathrm{T}$, after from this point the field is increased laterally again in symmetrically and continuous manner in two sides positive and negative of single circuit line until to reach a peak value of magnetic induction Bmax $=12.12 \mu \mathrm{T}$ under the side phase conductor, at a distance located between the lateral and central conductor about $x=9$ meters, then later reduces rapidly in symmetric way when one moves away from the conductors, to recorder at a distance of 75 meters, very far from the center line a minimum value is approximately $1.36 \mu \mathrm{T}$, about ten times lower than the maximum value found under the line. From previously mentioned above, On notice the calculated magnetic field strength which is within the acceptable range of the right of way (ROW) transmission line. On the other hand, It can also observed that the maximum value of magnetic field intensity under overhead power lines generally for frequency $50 \mathrm{~Hz}$ is below and not exceed the exposure limits values prescribed by the guidelines and internationals standards such as IRPA recommendations (International Association for Protection against Radiation), and CENELEC (European Committee for Standardization in Electrotechnique), are respectively $100 \mu \mathrm{T}$, and $500 \mu \mathrm{T}$.

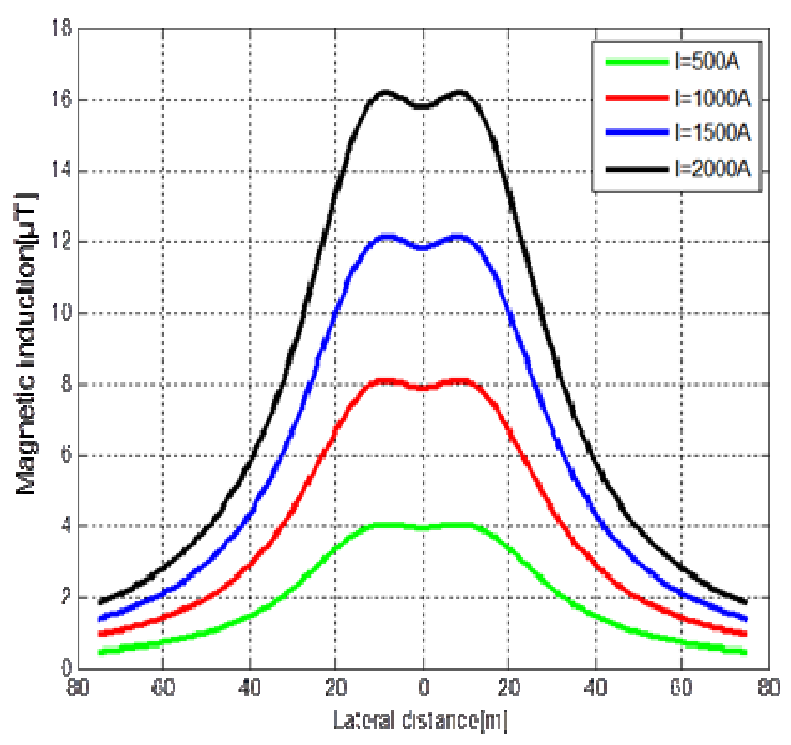

Fig. 5. The magnetic field intensity as a function of various values of loading current flowing in the phase conductors.

Fig. 5 shows the liaison between the magnetic field intensity and electric current flowing through the phase conductors, plots the lateral profile of the magnetic induction distribution at height $1 \mathrm{~m}$ above the ground as a function of different loading currents of phase conductors $I=500,1000,1500$, and $2000 \mathrm{~A}$. It is clear the maximum magnetic flux density values corresponding to this currents amplitude are 3.94, 7.88, 11.82 and $15.76 \mu \mathrm{T}$ respectively. It is very clear that as this curve is symmetric in the two sides positive and negative of single circuit line, it observe the currents magnitude increase, the magnetic field intensity increases until to reach the maximum values for all this four current values in the center distance under the middle phase conductors, then decreases rapidly with significative increase of the lateral distance, to recorder the minimum values when one moves away from the conductors, a very far for the center power line. On note the maximum magnetic flux density is found to be about $15.76 \mu \mathrm{T}$ corresponding to maximum current $\mathrm{I}=2000 \mathrm{~A}$. On the other hand, show the minimum magnetic flux density is found to be about $3.94 \mu \mathrm{T}$ corresponding to minimum current $\mathrm{I}=500 \mathrm{~A}$ flowing the conductors in all corridor right of way of the transmission line. The results obtained allow us saw significant linear relationship between the magnetic induction and current. More the current is higher, the magnetic field is intense. The magnetic field generated by overhead transmission line varies independently of 
the operating phase voltage, it is strong proportional to the loading current intensity that passes through them.

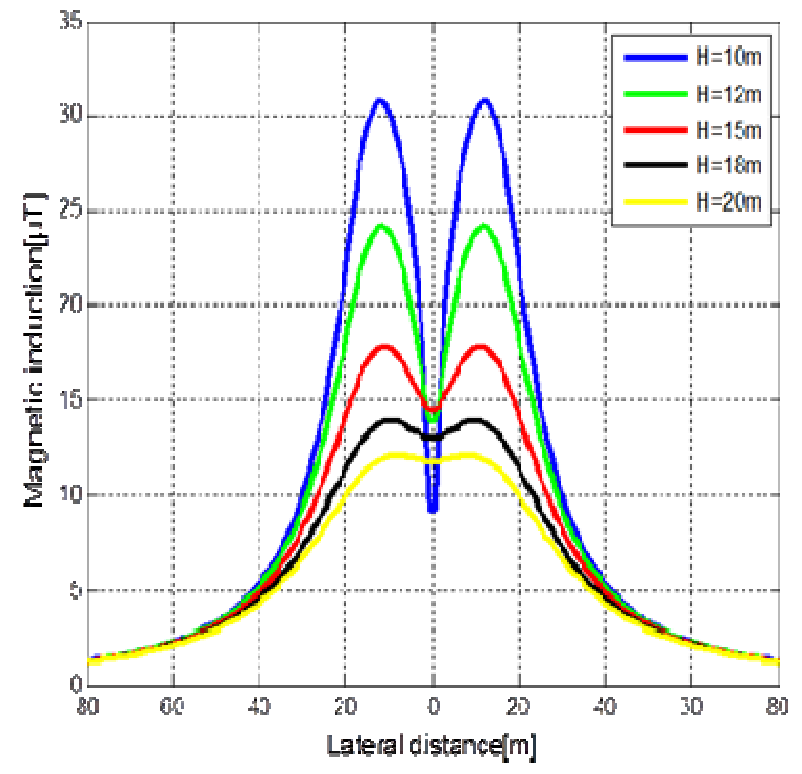

Fig. 6. The lateral profile variation of magnetic induction for various heights of phase conductors above the ground level.

Fig.6 plot the lateral profile variation of magnetic induction at $1 \mathrm{~m}$ above the ground as a function of various conductors heights $\mathrm{H}=10,12,15,18$ and $20 \mathrm{~m}$. It is very clear the maximum magnetic flux density values corresponding to these phase conductors heights are $30.75,24.17,14.55,13.04$ and $11.82 \mu \mathrm{T}$ respectively. As a shown in this curve, it is observed that as the heights of conductors increase, the magnetic field intensity decreases in symmetric manner in the two sides positive and negative of circuit line with a significative increase of the lateral distance, to reach the maximum values in the center distance under the middle phase conductors, then decreases rapidly to register the minimum values when one moves away from the conductors, a very far for the center power line in all this five phase conductors heights. It sees an opposite relationship between the magnetic induction and the height of conductors. Accordinate to this graph, on observe the maximum magnetic flux density is found to be about $30.75 \mu \mathrm{T}$ corresponding to minimum height of conductors $\mathrm{H}=10 \mathrm{~m}$. On the other hand, the minimum magnetic flux density is found to be about $11.82 \mu \mathrm{T}$ corresponding to maximum height of conductors $\mathrm{H}=20 \mathrm{~m}$ in all corridor right of way of the high voltage overhead transmission line. Increasing the line height is most effective parameter in the line design, which reduces the maximum field strength for all circuit lines configurations and phases arrangement. The changement of conductors height in the power line have great impact in the field intensity variation by increase or minimize the field stress under high voltage overhead power line, Therefore optimization or modification of the power line geometry in all configurations and phase conductors arrangement.

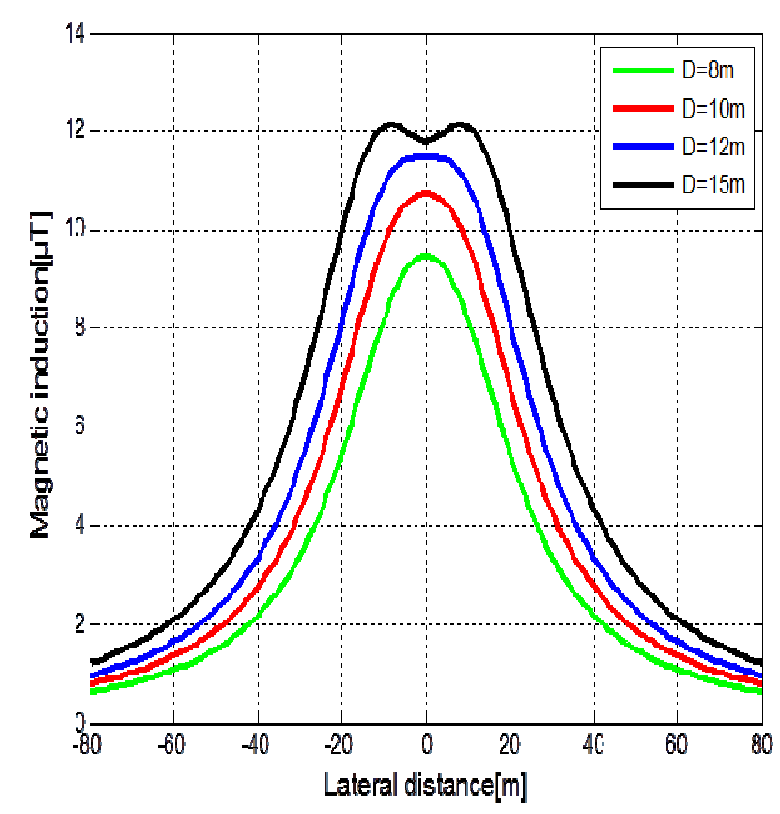

Fig. 7. The lateral profile variation of magnetic induction distribution for various phases spacing between conductors.

Fig.7 plot the lateral profile variation of magnetic induction at $1 \mathrm{~m}$ above the ground level as a function of various separation distances between the phase conductors $\mathrm{D}=8,10,12$, and $15 \mathrm{~m}$. It is very clear the maximum magnetic flux density values corresponding to these separation distances between phase conductors are $9.47,10.73,11.48$ and $11.82 \mu \mathrm{T}$ respectively. As a shown in this curve, it is observed that as the separation distances between phase conductors increase, the magnetic field intensity increases in continuous and symmetric manner in the two sides positive and negative of single circuit line, to reach the maximum values in the center distance under the middle phase conductors, then decreases rapidly with a significative increase of the lateral distance, to register the minimum values when one moves away from the conductors, a very far for the center power line in all this four phase spacing between conductors. Accordinate to this graph, on observe the maximum magnetic flux density is found to be about $11.82 \mu \mathrm{T}$ corresponding to maximum separation distance between phase conductors $\mathrm{D}=15 \mathrm{~m}$. On the other hand, the minimum magnetic flux density is found to be about $9.47 \mu \mathrm{T}$ corresponding to minimum separation distance between phase conductors $\mathrm{D}=8 \mathrm{~m}$. This variation is significative linearity relationship between them in all corridor right of way of the high voltage overhead transmission line, for all types of circuit lines configurations and phase conductors arrangement. So, the changement of separation distances between phase conductors have a great impact in the line design for increases or reduces the field strength, elsewhere the optimization of field intensity and modification case geometry under high voltage overhead power lines in each phases arrangement and circuit lines configuration, which is solution method for obtained the magnetic field minimization for overhead power transmission line. 


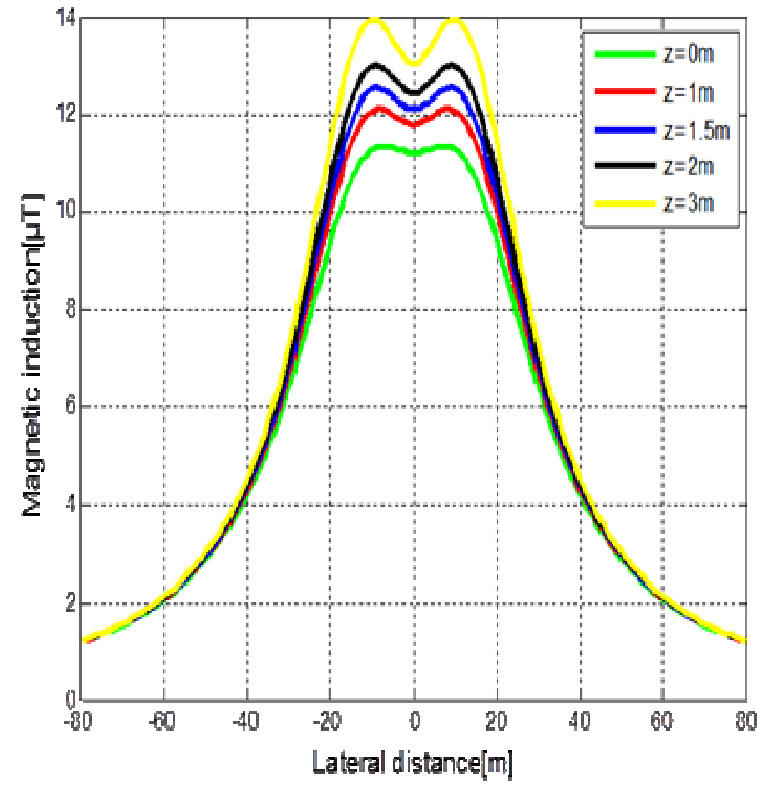

Fig. 8. The lateral profile variation of magnetic induction distribution for various observation point heights above earth.

Fig.8 plot the lateral profile variation of magnetic induction at $1 \mathrm{~m}$ above the ground as a function of various observation point heights above the ground (calculation point of the magnetic field ) $\mathrm{z}=0,1,1.5,2$ and $3 \mathrm{~m}$. It is very clear the maximum magnetic flux density values corresponding to these observation point heights are 11.23, 11.82, 12.13, 12.43 and $13.04 \mu \mathrm{T}$ respectively. As a shown in this curve it is clear that as the heights of observation point above the earth increase, the magnetic field intensity increases in continuous and symmetric manner in the two sides positive and negative of single circuit line, to reach the maximum values in the center distance under the middle phase conductors, then decreases rapidly with a significative increase of the lateral distance, to register the minimum values when one moves away from the conductors, a very far for the center power line in all this five heights of observation point above the ground level. Accordinate to this graph, on observe the maximum magnetic flux density is found to be about $13.04 \mu \mathrm{T}$ corresponding to maximum observation point height $\mathrm{z}=3 \mathrm{~m}$. On the other hand, the minimum magnetic flux density is found to be about $11.23 \mu \mathrm{T}$ corresponding to minimum observation point height $\mathrm{z}=0 \mathrm{~m}$ (ground level). Remarque from the foregoing, it we notice that this variation in the lateral profile of magnetic field generated by three-phase circuit line is significative linearity relationship between them in all corridor right of way of the high voltage overhead transmission line. Note according to the simulation results obtained that the magnetic induction values under and in the vicinity of high-voltage three-phase overhead power line, depending on the distance to several levels starting with the beginning level $0 \mathrm{~m}$ at the ground plane, therefore, on show clearly a two-dimensional 2D plot of the lateral profile of magnetic field distribution for this following levels as a function of the lateral distance of overhead power line for all types of circuit lines configurations and phase conductors arrangement.

Fig.9 illustrates the lateral profile of magnetic induction distribution in four different phases configuration of high voltage overhead power transmission line: horizontal, vertical, triangular, and inverted triangular circuit line at height $1 \mathrm{~m}$ from the ground level. On observe in this curve the magnetic field intensity increases in symmetrically and continuous manner in the two sides positive and negative of single circuit line, to reach the maximum values in the center distance under the middle phase conductors for all this four single circuit line configurations, then decreases rapidly for significative increase of the lateral distance of overhead power line, to reach the lower values a very far from the center line.

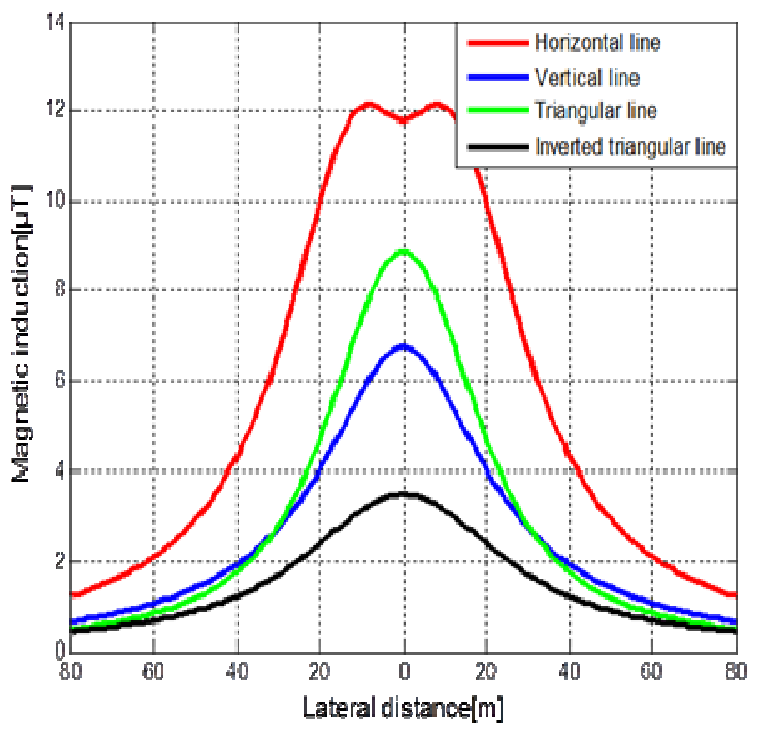

Fig. 9. Lateral profile of magnetic induction for different configurations of single circuit overhead transmission lines.

By comparing the values obtained, it can be observed that the magnetic induction is higher for the horizontal configuration at all points on the domain right of way transmission line. On the other hand, the calculated magnetic induction is lower for the inverted triangular configuration single circuit line in comparison with the magnetic induction values with other configurations (vertical, triangular), the last register the value $6.77 \mu \mathrm{T}$ for vertical configuration, in the addition the value $8.85 \mu \mathrm{T}$ for triangular configuration, respectively. As a shown in this curve, It is very clear that the horizontal flat configuration generates the greatest value of magnetic induction with a maximum value of $11.82 \mu \mathrm{T}$, although the inverted triangular configuration produces the lower values of magnetic induction with a maximum value of $3.48 \mu \mathrm{T}$ in the center configuration for the same current magnitude of 1500A. Elsewhere, from previously mentioned above, on notice this deviation in the lateral profile of magnetic field distribution at $1 \mathrm{~m}$ above the ground is due to difference in the height of phase conductors in each single circuit configuration. While 
the reverse triangular configuration should be the best solution when changing in the modification case of the basic geometry of circuit line for obtained the reduced values of the field intensity under the power line. So, the changement of phases arrangement have a great impact in the power line construction for increases or reduces the field strength, obtained the suitable phase conductors arrangement, Therefore, achieved the optimization and minimization of the field intensity under and in the vicinity of high voltage overhead transmission lines.

\section{Conclusions}

In this article we used the image method for calculation the lateral profile distribution of magnetic field intensity generated by the high voltage three-phase overhead power line arranged in single circuit horizontal configuration at height $1 \mathrm{~m}$ above the ground level. Depending on the simulation results, we see the magnetic induction values are maximum in the middle point of the power line at a center distance of phase conductors, then decrease quite rapidly in symmetrically manner for significative increase of the lateral distance, to reach the lower values when one moves away from the conductors at a center distance far from power line. There are many factors that affect in the magnetic induction values produced under and in the vicinity of the high voltage overhead transmission line, it mainly depends on the geometry of circuit line, that is to say the arrangement of phases conductors, the current magnitude carry through the conductors, the height of phase conductors above the ground, the separation distance between phase conductors, and the observation point height above earth where the magnetic field is calculated. Based in the simulation results it is very clear the magnetic flux density is higher for horizontal configuration than the other configurations triangular and vertical single circuit line, but the magnetic flux density for reverse triangular configuration is lesser compared to the other conductors configurations. The calculation maximum magnetic induction values underneath high voltage overhead power lines with the same current amplitude of $1500 \mathrm{~A}$ is minimum and does not exceed the value $20 \mu \mathrm{T}$, which is five times lower of the exposure limit value established by international standard ICNIRP for the general public $100 \mu \mathrm{T}$. in the future research we find simple and fast methods for accurate fastly the magnetic field of high voltage overhead power line for any complex geometry circuit power line, therefore minimize, and negliged the errors caused in the temporal period or the tools in test review. This system enables the simulation of the magnetic field created by the majority of overhead lines and presents a series of advantages such as the ease of being able to study different types of lines, as well as the possibility of predicting the magnetic field that projected lines will generate. The simulation code analysing and understanding thoroughly the steps involved in the computation of magnetic field. Results from simulations through applying the method explained in this work have been precisely for observe the magnetic field profile.

\section{Appendix}

Table 2. Lists of different abbreviations.

\begin{tabular}{|c|c|}
\hline Abbreviation & Name \\
\hline$\mu \mathrm{T}$ & Micro tesla \\
\hline $\mathrm{kV}$ & Kilo volt \\
\hline $\mathrm{A}$ & Ampere \\
\hline $\mathrm{ICNIRP}$ & $\begin{array}{l}\text { International Commission on } \\
\text { Pon-Ionizing Radiation } \\
\text { Protection }\end{array}$ \\
\hline $\mathrm{Hz}$ & Hertez \\
\hline $\mathrm{m}$ & Meter \\
\hline
\end{tabular}

\section{References}

1. A. Cigre, Electrical and magnetic fields generated by transmission networks, example of calculation of electromagnetic disturbances by the CIGRE method, International Conference of Large High-voltage Electrical Networks, Paris Edition Dunod, 21-43 (1983)

2. G. Kulkarni, W.Z. Gandhare, Proximity effects of high voltage transmission lines on humans, ACEEE Int. J. on Electrical and Power Engineering 3, 1, 2832 (2012)

3. R. Radwan, M. Abdel-Salam, A.B. Mahdy, M. Samy, Laboratory Validation of Calculations of Magnetic Field Mitigation Underneath Transmission Lines Using Passive and Active Shield Wires, Innovative Systems Design and Engineering 2, 4, 218-232 (2011)

4. J. C. Bravo-Rodríguez, J .C. del-Pino-López, and P.A. Cruz-Romero, Survey on Optimization Techniques Applied to Magnetic Field Mitigation in Power System, Escuela Politécnica Superior, Universidad de Sevilla. Energies 12, 7 p. 1332 , https://doi:10.3390/en12071332 (2019)

5. Jamal M. Ehtaiba, Sayeh M. Elhabashi, Magnetic Field Around the New 400kV OH Power

Transmission Lines In Libya, Proceedings of the Wseas International Conference on environment, medicine and health sciences, January 2010

6. W. Tourab, A. Babouri, Measurement and modeling of personal exposure to the electric and magnetic fields in the vicinity of high voltage power lines, Safety and Health at Work 7, 10, 102-110, (2016)

7. R. Amiri, H. Hadi, M. Marich, The influence of sag in the electric field calculation around high voltage overhead transmission lines, In:2006 IEEE 
Conference on Electrical Insulation and Dielectric Phenomena. IEEE, 206-209, (2006)

8. R. Deltuva, R. Lovrić, Distribution of magnetic field in $400 \mathrm{kV}$ double-circuit transmission lines, Applied Sciences 10, 9, p. 3266

9. S. Vujević, D. Lovrić, P. Sarajčev, Comparison of $2 D$ algorithms for the computation of power line electric and magnetic fields, European Transactions on Electrical Power 21, 1, 505-521 (2011)

10. K Erenturk, MATLAB-based GUIs for fuzzy logic controller design and applications to PMDC motor and AVR control, Computer Applications in Engineering Education 13, 1, 10-25 (2005)

11. J. R. Riba Ruiz, A. Garcia Espinosa, J. A. Ortega, Validation of the parametric model of a DC contactor using Matlab-Simulink, Computer Applications in Engineering Education, Published online in Wiley Inter Science, DOI: 10.1002/cae.20315 19, 2, 337-346 (2011)

12. C. Hamilton, Using MATLAB to advance the robotics laboratory, Computer Applications in Engineering Education 15, 3, 205-213 (2007)

13. M. C. M. Teixeira, E. Assunção, M. R. Covacic, Proportional controllers: Direct method for stability analysis and MATLAB implementation, IEEE Transactions on Education 50, 1, 74-78 (2007)

14. C. Garrido, A. F. Otero, J. Cidras, Low-frequency magnetic fields from electrical appliances and power lines, IEEE Transactions on Power Delivery 18, 4, 1310-1319 (2003)

15. R. G. Olsen, T. A. Pankaskie, On the exact, carson and image theories for wires at or above the earth's interface, IEEE Transactions on Power Apparatus and Systems PAS-102, 4, 769-778 (1983)

16. H. M. Ismail, Characteristics of the magnetic field under hybrid ac/dc high voltage transmission lines, electric power systems Research 79, 1, 1-7 (2009)

17. P. A. Tipler, Physics for Scientifics and Engineers, 4th edition.W.H. Freeman and Company, Worth Publishers, New York, 892-893 (1990)

18. P. R. Bannister, Image theory results for the mutual impedance of crossing earth return circuits, IEEE Transactions on Electromagnetic Compatibility 4, 158-160 (1973)

19. K. Budnik, W. Machczyński, Contribution to studies on calculation of the magnetic field under power lines, European Transactions on Electrical Power 16, 4, 345-364 (2006)

20. M. Darveniza, A practical extension of Rusck's formula for maximum lightning-induced voltages that accounts for ground resistivity, IEEE Transactions on Power Delivery 22, 1, 605-612 (2006)

21. E. Resistivity, ANSI/IEEE Std 81, An American National Standard IEEE Guide for Measuring Earth Resistivity, Ground Impedance, and Earth Surface Potentials of a Ground System (1983)
22. D. Yao, B. Li, J. Deng, D. Huang, X. Wu, Power frequency magnetic field of heavy current transmit electricity lines based on simulation current method, In Proceedings 2008 World Automation Congress. IEEE, 1-4 (2008)

23. J.R Riba Ruiz, A. Garcia Espinosa, Magnetic field generated by sagging conductors of overhead power lines, Computer Applications in Engineering Education 19, 4, 787-794 (2009) 